\title{
EFFECT OF DIFFERENT LIGHT CURING INTENSITIES ON CAVITY WALL ADAPTATION OF NANOFILLED RESIN COMPOSITE USING TRANSTOOTH CURING TECHNIQUE: AN IN-VITRO STUDY
}

\author{
Shereen Hafez*, Ahmed El Zohairy* and Amir Hafez**
}

\begin{abstract}
Aim: to evaluate the cavity wall adaptation of nanofilled resin composite cured with different LED light intensities and with different thickness of buccal wall after thermocycling using environmental scanning electron microscope.

Materials and methods: the materials used for this study was nanofilled resin composite material (Filtex Z350XT, 3M, ESPE) shade A3with its corresponding bonding system (single bond universal, 3M, ESPE). A total of 10 sound extracted human maxillary premolar teeth were used in this study. The teeth were divided into 2 main groups of 5 teeth each according to curing light intensities; either standard LED curing mode $\left(800 \mathrm{~mW} / \mathrm{cm}^{2}\right)$ for $30 \mathrm{sec}$ or high intensity LED curing mode $\left(1400 \mathrm{~mW} / \mathrm{cm}^{2}\right)$ for $10 \mathrm{sec}$. In each tooth, 2 cavity preparations were performed; moderate cavity preparation width (1/3 intercuspal distance) in the mesial aspect of each tooth and wide cavity preparation (1/2 intercuspal distance) in the distal aspect. All specimens were light cured using transtooth curing technique from the buccal and lingual surfaces. All specimens were subjected to thermocycling protocol. All specimens were subjected to the quantitative margin analysis, examined using environmental scanning electron microscope (ESEM). Two ways analysis of variance (ANOVA) test was used to study the interaction of both variables. The significance level was set at $\mathrm{p}<0.05$.
\end{abstract}

Results: Two ways ANOVA test revealed that the interaction of both variables was statistically significant $(\mathrm{p}=0.00)$. Comparing both intensities, a higher mean value was recorded in high intensity $(6.57 \pm 1.29)$, with a significant difference $(\mathrm{p}=0.00)$. Comparing $1 / 2 \mathrm{ICD}$ and $1 / 3 \mathrm{ICD}$, a higher mean value was recorded in $1 / 3 \mathrm{ICD}(6.52 \pm 2.19)$, with a significant difference $(\mathrm{p}=0.00)$.

Conclusions: The wall thickness greatly affects the cavity wall adaptation in transtooth curing technique.Applying High intensity irradiation technique compromise margin quality and marginal seal of resin composite restoration.

KEY WORDS: high LED light intensiy, transtooth curing, cavity wall adaptation, interfacial analysis, nanofilled composite.

* Ass. Professor of Conservative Dentistry, Faculty of Dentistry, Cairo University

** Lecturer of Conservative Dentistry, Faculty of Dentistry, Cairo University 


\section{INTRODUCTION}

The polymerization shrinkage of resin composites lead to contraction gaps between the restoration and cavity wall. Such contraction gaps may lead to post-operative sensitivity, pain on biting, marginal staining, and recurrent caries ${ }^{[1]}$. In addition to marginal enamel cracks that occur when the bond strength to tooth structure exceed contraction stress and appear as white crack line in enamel ${ }^{[2]}$.

There are several ways to overcome the curing stresses generated by light-cured resin composites such as varying the photo-activation method; curing the resin composites at low light intensity have been shown to have a better marginal adaptation; due to the slower rate of conversion that maintains a longer pre-gel phase, allowing for a better flow of the material, which decreases contraction stress in the filling material. However, this low intensity may affect the surface hardness and may be insufficient for ensuring mechanical stability. ${ }^{[3]}$

Recently, high intensity light curing device are used to shorten the curing time and polymerizing the deeper parts of resin composite fillings. However, this technique employed may lead to variations in the physical and mechanical properties of these materials, and also may lead to a higher frequency of marginal and wall gap formation by creating more contraction forces and finally marginal failure. ${ }^{[4]}$

When a composite was cured with initial lowintensity light followed by high-intensity light, excellent marginal sealing and cavity adaptation were achieved due to rearrangement of the polymeric chains during the initial polymerization phase. ${ }^{[2]}$ Pre-polymerization at low intensity, followed by the final cure at high intensity, can allow for the flow of resin composite during setting. This method can reduce the width and length of marginal gaps without interfering with the physical properties of the restorations. ${ }^{[3]}$

Transtooth curing technique has been introduced as guided polymerization technique for restoration of prepared class II cavity to provide better adaptation of resin composite restoration. In this technique, the Light transmitted through the tooth structure initially polymerizes the most critical areas along the interfaces, establishing adequate bonding before polymerization shrinkage of the inner bulk interferes. ${ }^{[5,6]}$

New methods of polymerization with varying intensities and curing times are on the market; therefore, it is necessary to analyze the effectiveness in the control of marginal adaptation and the quality of polymerization. This study was carried out to evaluate the cavity wall adaptation of nanofilled resin composite cured with different LED light intensities and with different thickness of buccal wall after thermocycling using environmental scanning electron microscope.

The null hypothesis of this study is that no significant effect on cavity wall adaption of resin composite restoration either cured with high or low intensity transtooth curing method through different thickness of buccal wall.

\section{MATERIALS AND METHODS}

The materials used for this study was nanofilled resin composite material (Filtex Z350XT, 3M, ESPE, St Paul, MN, USA Batch\#N731284) shade A3with its corresponding bonding system (single bond universal, 3M, ESPE). The resin composite contains, bis-GMA, UDMA, TEGDMA, and bis-EMA resins according the manufacturer. PEGDMA has been substituted for a portion of the TEGDMA resin to moderate the shrinkage. The fillers are a combination of non-agglomerated/nonaggregated $20 \mathrm{~nm}$ silica filler, non-agglomerated/ non-aggregated 4 to $11 \mathrm{~nm}$ zirconia filler, and aggregated zirconia/silica cluster filler (comprised of $20 \mathrm{~nm}$ silica and 4 to $11 \mathrm{~nm}$ zirconia particles). The inorganic filler loading is about $72.5 \%$ by weight (55.6\% by volume). For photo-activation, LED curing unit (Dr's light AT, Good doctors co.ltd. korea) with two different light intensities were used in this study. 


\section{Sample preparation:}

A total of 10 sound extracted human maxillary premolar teeth were used in this study. The selected teeth had an average occluso-gingival height of teeth $4.3 \mathrm{~mm}$ and their average bucco-lingual dimension was $8.6-10.5 \mathrm{~mm}$ that was checked using a digital caliper (Ruifeng Foreign, China). Teeth with a minimum occluso-gingival height of were selected. The teeth were divided into 2 main groups of 5 teeth each according to curing light intensities; either standard LED curing mode $\left(800 \mathrm{~mW} / \mathrm{cm}^{2}\right)$ for 30 $\mathrm{sec}$ or high intensity LED curing mode $(1400 \mathrm{~mW} /$ $\mathrm{cm}^{2}$ ) for $10 \mathrm{sec}$. In each tooth, 2 cavity preparations were performed; moderate cavity preparation width one third intercuspal distance (1/3 ICD) in the mesial aspect of each tooth and wide cavity preparation with half intercuspal distance (1/2 ICD) in the distal aspect. Thus, a total of 20 cavity preparations were performed. All specimens were light cured using transtooth curing technique from the buccal and lingual surfaces.

Two standardized, compound class II cavity preparations were prepared on the proximal surfaces of all teeth using no. 245 carbide bur (Healthico, USA) in a high speed hand-piece (PANA MAX, NSK, Japan) under copious amount of water. The carbide bur was changed every 10 preparations. Cavity preparation dimensions were then measured using a digital caliper and the outline was drawn. The cavity preparation dimensions were approximately standardized to $4 \mathrm{~mm}$ occluso-gingival height and $1.5 \mathrm{~mm}$ axial wall depth. Regarding the width, the mesial cavity preparation was standardized to 3 $\mathrm{mm}$ bucco-lingual width representing the moderate cavity width, while the distal cavity preparation was $4 \mathrm{~mm}$ for wide cavity preparation.

\section{Filling and curing technique:}

Each tooth with mesial and distal prepared cavities was placed in dental study model (El Banna, Egypt) with proper mesial and distal contact with adjacent ivory teeth. A pre-contoured Sectional matrix, standard kit (Palodent Sectional Matrix
System, Dentsply, USA) was used. Two standard size matrices with BiTine round rings were placed mesially and distally with proper wooden wedge size. All prepared cavities were restored using nanofilled resin composite material (Filtex Z350XT, $3 \mathrm{M}$, ESPE). The same bonding protocol was performed, according to manufacturer's instructions, for all groups. The adhesive system (single bond universal, 3M, ESPE) was applied using disposible microbrush, agitated for $10 \mathrm{sec}$ and then the adhesive was thinned with gentle air stream using light pressure for 5 seconds and polymerized using a LED curing unit $\left(800 \mathrm{~mW} / \mathrm{cm}^{2}\right)$ for 20 seconds, with perpendicular angulation at zero distance. The resin composite was placed incremently in both cavities using a gold-plated composite applicator (Miltax, Germany). The resin composite material was then light cured using LED light curing unit according to the previously described testing groups using different predetermined light intensities. Before each curing cycle, the light irradiance was checked using the built-in radiometer. For Transtooth curing technique, before light curing, the matrix ring retainers were removed and the band ends were slightly displaced such that the curing tip is in direct contact with the tooth and one light curing cycle was applied from buccal and lingual transtooth direction.

\section{Thermocycling procedure:}

All specimens were subjected to thermocycling protocol using Robota automated thermal cycle device (BILGE, Turkey); the number of cycles used was 500 cycle. The low-temperature point was $5^{\circ} \mathrm{C}$ and the high-temperature point was $55^{\circ} \mathrm{C}$. Dwell times were 25 seconds in each water bath with a lag time 10 seconds. ${ }^{[2]}$

\section{Assessment of tooth-restoration interface:}

All specimens were subjected to the quantitative margin analysis, examined using environmental scanning electron microscope (ESEM). The samples were sectioned longitudinally in two halves 
by passing through restoration center then the cut surfaces of specimens were finished and polished, then immersed in $6 \mathrm{~mol} / \mathrm{L}$ hydrochloric acid (HCL) for 30 seconds to remove polishing smear layer and bring the resin-dentin interface into relief through partial dissolution of dentine then rinsed with distilled water and immersed in $1 \%$ sodium hypoclorite (NAOCL) for $10 \mathrm{~min}$ to remove any collagen fibrils that were not protected by resin infiltration, and then rinsed again with distilled water. Finally specimens were immersed in alcohol for 10 minutes and left to dry before examination. The interfacial gaps at three different levels were measured in each photomicrograph and the mean was taken, the values were first obtained in $(\mathrm{mm})$ and were then converted into micron $(\mu \mathrm{m})$ fig. $(2-5){ }^{[7,8]}$

\section{Statistical analysis}

Numerical data were explored for normality using Kolmogorov-Smirnov test of normality. Normally distributed (parametric data), were presented as mean, standard deviation (SD), standard error and confidence intervals and were compared between groups using independent $t$ test. Two ways analysis of variance (ANOVA) test was used to study the interaction of both variables. The significance level was set at $p<0.05$. Statistical analysis was performed with SPSS 18.0 (Statistical Package for Scientific Studies, SPSS, Inc., Chicago, IL, USA) for Windows.

\section{Comparison between 1/2 ICD and 1/3 ICD with- in the same intensity}

Using high intensity, a higher mean value was recorded in $1 / 3$ ICD $(9.08 \pm 1.53)$ in comparison to $1 / 2$ ICD (4.05 \pm 1.08$)$, with a significant difference $(\mathrm{p}=0.00)$. Using low intensity, a higher mean value was recorded in 1/3 ICD (3.96 \pm 0.71$)$ in comparison to $1 / 2$ ICD $(2.09 \pm .43)$, with a significant difference $(\mathrm{p}=0.00),($ Table 1, Fig. 1)

\section{Comparison between high and low intensity}

Using 1/2ICD, a higher mean value was recorded in high intensity $(4.05 \pm 1.08)$ in comparison to low intensity (2.09 \pm 0.43$)$, with a significant difference $(\mathrm{p}=0.00)$. Using $1 / 3 \mathrm{ICD}$, a higher mean value was recorded in high intensity $(9.08 \pm 1.53)$, in comparison to low intensity $(3.96 \pm 0.71)$, with a significant difference $(\mathrm{p}=0.00),($ Table 2, Fig. 1)

\section{Interaction of variables}

Two ways ANOVA test revealed that the interaction of both variables was statistically significant $(\mathrm{p}=0.00)$. Comparing both intensities, a higher mean value was recorded in high intensity $(6.57 \pm 1.29)$, with a significant difference $(p=0.00)$. Comparing 1/2ICD and 1/3ICD, a higher mean value was recorded in $1 / 3 \mathrm{ICD}(6.52 \pm 2.19)$, with a significant difference $(\mathrm{p}=0.00)$.

Table (1) Comparison between 1/2 ICD and 1/3 ICD within the same intensity (independent $\mathrm{t}$ test)

\begin{tabular}{|c|c|c|c|c|c|c|c|c|c|c|}
\hline & \multirow[b]{2}{*}{ Groups } & \multirow[b]{2}{*}{ Mean } & \multirow[b]{2}{*}{$\begin{array}{l}\text { Std. } \\
\text { Dev }\end{array}$} & \multirow[b]{2}{*}{$\begin{array}{c}\text { Std. Error } \\
\text { Mean }\end{array}$} & \multicolumn{4}{|c|}{ Difference } & \multirow[b]{2}{*}{$\mathrm{t}$} & \multirow[b]{2}{*}{$P$} \\
\hline & & & & & Mean & $\begin{array}{l}\text { Std. } \\
\text { Error }\end{array}$ & $\begin{array}{c}\text { 95\% C.I. } \\
\text { Lower }\end{array}$ & $\begin{array}{l}\text { 95\% C.I } \\
\text { Upper }\end{array}$ & & \\
\hline \multirow{2}{*}{ High intensity } & $1 / 2 \mathrm{ICD}$ & 4.05 & 1.08 & .34 & \multirow{2}{*}{-5.03} & \multirow{2}{*}{.59} & \multirow{2}{*}{-6.29} & \multirow{2}{*}{-3.78} & \multirow{2}{*}{-8.49} & \multirow{2}{*}{$.000^{*}$} \\
\hline & $1 / 3 \mathrm{ICD}$ & 9.08 & 1.53 & .48 & & & & & & \\
\hline \multirow{2}{*}{ Low intensity } & $1 / 2 \mathrm{ICD}$ & 2.09 & .43 & .14 & \multirow{2}{*}{-1.87} & \multirow{2}{*}{.26} & \multirow{2}{*}{-2.44} & \multirow{2}{*}{-1.31} & \multirow{2}{*}{-7.10} & \multirow{2}{*}{$.000^{*}$} \\
\hline & $1 / 3 \mathrm{ICD}$ & 3.96 & .71 & .23 & & & & & & \\
\hline
\end{tabular}

Significance level $p<0.05$, *significant 
TABLE (2) Comparison between low and high intensity (independent $t$ test)

\begin{tabular}{|c|c|c|c|c|c|c|c|c|c|c|}
\hline & \multirow[b]{2}{*}{ Intensity } & \multirow[b]{2}{*}{ Mean } & \multirow[b]{2}{*}{ Std. Dev } & \multirow[b]{2}{*}{$\begin{array}{c}\text { Std. Error } \\
\text { Mean }\end{array}$} & \multicolumn{4}{|c|}{ Difference } & \multirow[b]{2}{*}{$\mathrm{t}$} & \multirow[b]{2}{*}{$\mathrm{P}$} \\
\hline & & & & & Mean & $\begin{array}{l}\text { Std. } \\
\text { Error }\end{array}$ & $\begin{array}{c}\text { 95\% C.I. } \\
\text { Lower }\end{array}$ & $\begin{array}{c}\text { 95\% C.I } \\
\text { Upper }\end{array}$ & & \\
\hline $1 / 2 \mathrm{ICD}$ & $\begin{array}{l}\text { High intensity } \\
\text { Low intensity }\end{array}$ & $\begin{array}{l}4.05 \\
2.09\end{array}$ & $\begin{array}{l}1.08 \\
.43\end{array}$ & $\begin{array}{l}.34 \\
.14\end{array}$ & 1.96 & .37 & 1.16 & 2.77 & 5.32 & $.000 *$ \\
\hline $1 / 3 \mathrm{ICD}$ & $\begin{array}{l}\text { High intensity } \\
\text { Low intensity }\end{array}$ & $\begin{array}{l}9.08 \\
3.96\end{array}$ & $\begin{array}{l}1.53 \\
.71\end{array}$ & $\begin{array}{l}.48 \\
.23\end{array}$ & 5.12 & .53 & 3.97 & 6.28 & 9.6 & $.000 *$ \\
\hline
\end{tabular}

Significance level $p<0.05$, *significant

TABLE (3) Results of two ways ANOVA test for interaction of both variables

\begin{tabular}{|c|c|c|c|c|c|}
\hline Grouping & Mean & Std. Dev & $\begin{array}{l}\text { 95\% Confidence } \\
\text { Interval }\end{array}$ & & $\mathrm{P}$ \\
\hline & & & Lower Bound & Upper Bound & \\
\hline High intensity & 6.565 & 1.29 & 6.099 & 7.030 & $.000 *$ \\
\hline Low intensity & 3.023 & 1.07 & 2.557 & 3.488 & \\
\hline $1 / 2 \mathrm{ICD}$ & 3.068 & 1.05 & 2.602 & 3.533 & $.000 *$ \\
\hline $1 / 3 \mathrm{ICD}$ & 6.520 & 2.19 & 6.054 & 6.985 & \\
\hline Interaction of both variables & & & & & $.000 *$ \\
\hline
\end{tabular}

Significance level $p<0.05$, *significant

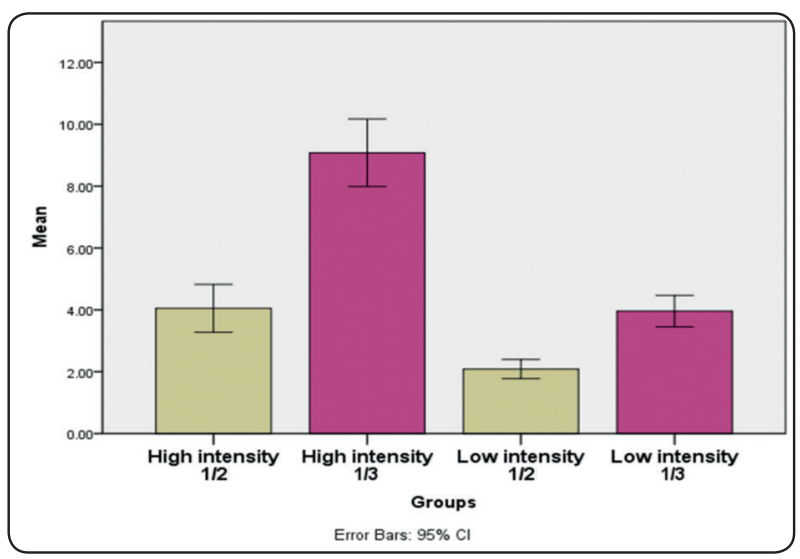

Fig. (1) Bar chart showing mean value of measurements in all subgroups

\section{Interfacial analysis:}

Fig 2-5 show A representative photomicrography samples of Resin dentin interfaces of each group were analyzed using a Scanning Electron Microscope (SEM) at $1000 \times$ magnificatio 


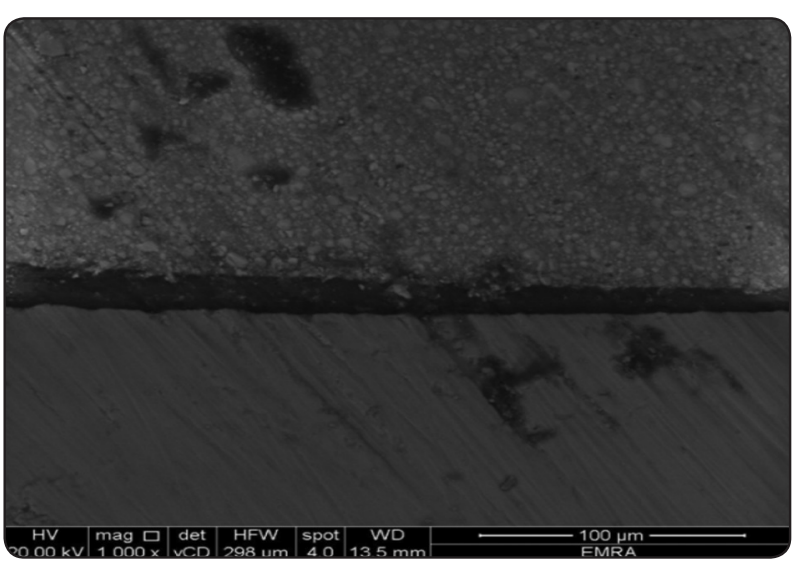

Fig. (2) Photomicrographs of high intensity in $1 / 3$ ICD

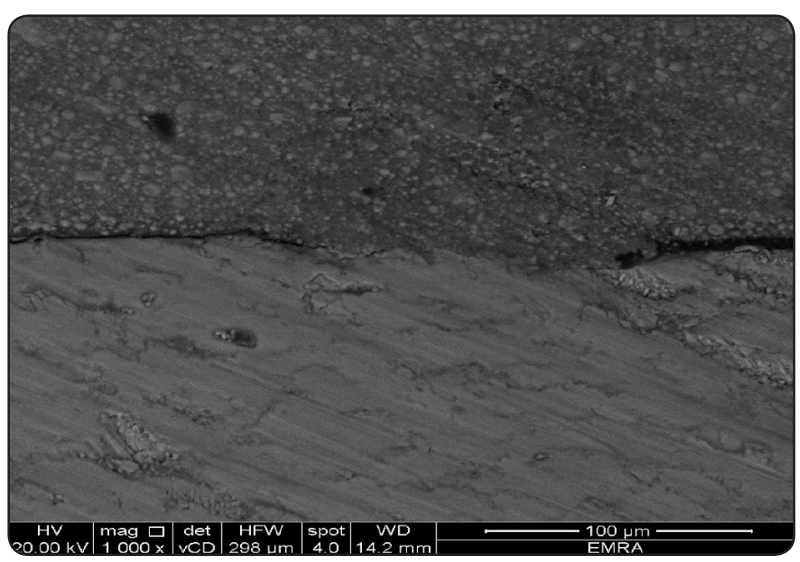

Fig. (4) Photomicrographs of low intensity in 1/3 ICD

\section{DISCUSSION}

Resins Composites become the most popular restorative materials due to its esthetics and good clinical performance. However marginal adaptation remains the most common problem with the resin composite restoration. This due to dimensional changes in the materials involves polymerization shrinkage, differences in coefficient of thermal expansion and hygroscopic absorption of the materials that result in inadequate margin adaptation of restorative material and gap formation at the tooth-material interface. ${ }^{[1]}$

Cavity adaptation of the composite restoration was dependent on the interaction between polymerization contraction stress and the efficacy

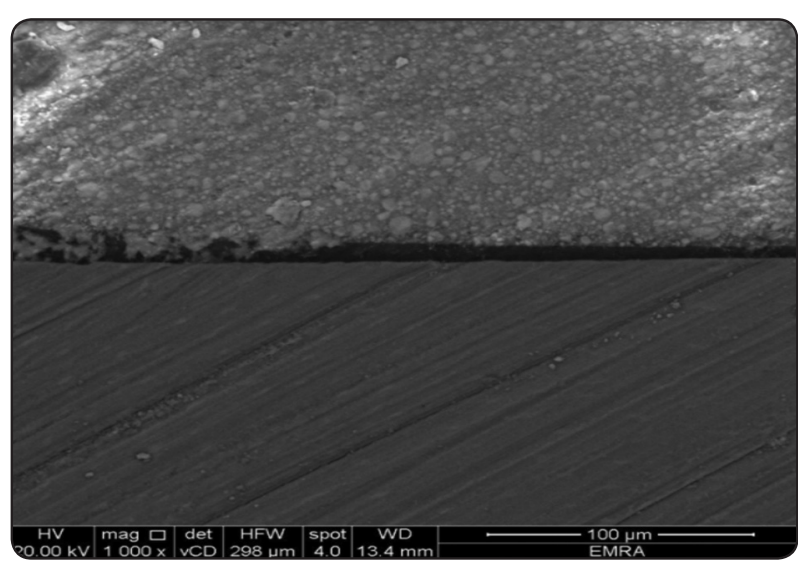

Fig. (3) Photomicrographs of high intensity in 1/2 ICD

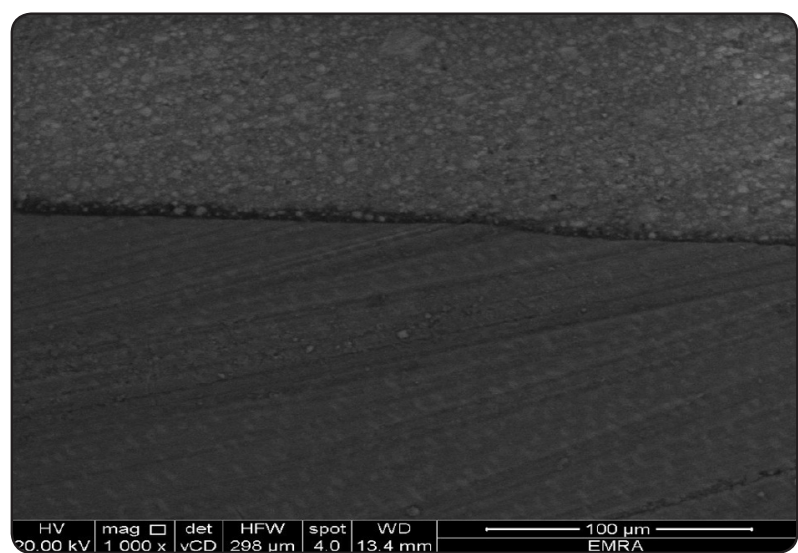

Fig. (5) Photomicrographs of low intensity in 1/2 ICD

of the bonding system employed. ${ }^{[1]}$ Many factors affect the development of contraction stress in dental composites. These can be separated into material formulation factors and material polymerization factors. ${ }^{[9]}$ Bonding the composite resin to the cavity walls generates shrinkage stress on the bonding interfaces. If this stress exceeds the bond strength between the dental substrate and the adhesive system, a contraction gap will be formed that jeopardizing the restoration's longevity. ${ }^{[8]}$

Little published information exists about the effectiveness of transtooth-illumination techniques on cavity wall adaptation of resin composite, so this study was carried out to evaluate the cavity wall adaptation of nanofilled resin composite cured with 
different LED light intensities and with different thickness of buccal wall after thermocycling using environmental scanning electron microscope.

In this study, every effort was exerted to standardize the methodology and simulate the clinical conditions. A standardized box-shaped preparation was made on mesial and distal of each tooth for the moderate width (1/3 ICD) and wide (1/2 ICD) cavity preparations. In addition, a standardized cavity preparation dimension would result in a standard amount of composite restoration to be polymerized. ${ }^{[6]}$

The performance of the transtooth-illumination technique could be a result of the ability of light to penetrate enamel and dentin walls and cure the inner portion of a restoration, and this return to the amount of light energy applied with this technique, as the current-generation curing lights with high outputs that could cure adequately through the tooth structures. ${ }^{[5]}$ So in this study transtooth curing technique was applied by two light intensity either standard LED curing mode $\left(800 \mathrm{~mW} / \mathrm{cm}^{2}\right)$ for 30 $\mathrm{sec}$ or high intensity LED curing mode $(1400 \mathrm{~mW} /$ $\mathrm{cm}^{2}$ ) for $10 \mathrm{sec}$.

Thermocycling is an important procedure for testing the sealing ability of restorative material, due to the thermally induced stresses resulted from the mismatch between the coefficients of thermal expansion for the restorative material and the natural tooth structure, may lead to gap formation and Microleakage. ${ }^{[2]}$ So in this study specimens were subjected to 500 thermal cycles between $5^{\circ} \mathrm{C}$ and $55^{\circ} \mathrm{C}$ with a dwell time of 25 seconds in each water bath in order to simulate aging conditions.

The result of current study reject the null hypothesis as the tested resin composite material (Filtek $^{\text {TM }}$ Z350) cured by transtooth technique with low intensity exhibited statistically the highly significant decrease in marginal gaps width along the interface after thermomechanical load cycling as compared with high intensity one, same as for the narrow buccal wall thickness of $1 / 2 \mathrm{ICD}$ cavity recorded significant reduction in marginal gap width compared with the thick buccal of 1/3 ICD cavity.

This was explained by Yaroub et al ${ }^{[8]}$ as the distance of light source from the material which is higher at the proximal box with $1 / 3$ ICD compared to $1 / 2$ ICD cavity; which affect the irradiance of the light reaching resin composite resin and reducing the percentage of degree of conversion. Low degree of monomer conversion might cause unconverted double bonds, making the resin more susceptible to degradation at the tooth-restoration interface.

It has been pointed out that reduced light intensity improves marginal adaptation of photo-polymerized resin composites. Photo-polymerization by low intensity may allow most of the polymerization contraction to be completed during the initial viscous phase of composite polymerization, and reduce polymerizing stress which causes debonding at the composite/tooth interface. ${ }^{[2]}$ However, the high intensity more frequently lead to gap formations at tooth/restoration interface due to its negative effect on the development of stress during the polymerization also increases in the rate of the polymerization initiation lead to reductions in the average length of the polymer chains and increases the rate of water uptake. ${ }^{[2,9]}$

In other word Hardan et al ${ }^{[9]}$ mentioned that the polymerization of the first composite layer with an low light intensity result in lengthen the phase of the early setting of the composite, so that polymerization shrinkage takes place when the molecules still have the possibility of taking a new direction to compensate the internal stress, thus the contraction stress developed during the final setting is decreased, and can be resisted by the adhesive forces of the bonding.

This was contradicted by Shinkai et al ${ }^{[1]}$ who found no significant differences between the cavity adaptation of resin composite restorations polymerized with light intensities higher than 
$1100 \mathrm{~mW} / \mathrm{cm}^{2}$ and those polymerized with 400 $\mathrm{mW} / \mathrm{cm}^{2}$ light intensity, and they explained that the compensation for the polymerization contraction stress by effectiveness of the bonding system used and the expansion of polymerized resin composite by water absorption. The results may be opposite when different adhesive systems and resin composite were used and this considered a limitation of our study as only one product from one manufacturer were used. The results on marginal quality may have been different if other materials, especially other bonding systems, had been used.

Furthermore bortolotto et al ${ }^{[10]}$ stated that as dentin is a more elastic substrate, the contraction stresses can be relieved and absorbed by the inherent elasticity of dentin, this explaining why no significant differences between their test groups were observed on dentin marginal adaptation

Additionally Yoshikawa et al ${ }^{[2]}$ showed that the use of a low initial light intensity for $10 \mathrm{sec}$ followed by high intensity light for $5 \mathrm{sec}$ provides the best adaptation of resin composite to cavity walls. This "soft-start polymerization" method may reduce polymerization stresses by delaying the hardening of the resin composite and allow more time for relief of polymerization contraction stress that result in composite restorations with improved marginal integrity without weakening the mechanical properties.

\section{CONCLUSIONS}

Within the limits of this study, the following conclusions can be derived:

1) The wall thickness greatly affects the cavity wall adaptation in transtooth curing technique.

2) Applying High intensity irradiation technique compromise margin quality and marginal seal of resin composite restoration.

\section{REFERENCES}

1. Shinkai, K., S. Suzuki, and Y. Katoh, Effect of high light intensity on cavity wall adaptation of a resin composite with a self-etching primer system. Journal of Biomedical Materials Research Part B: Applied Biomaterials, 2006. 79B(2): p. 420-424.

2. Yoshikawa, T., et al., Effects of light curing method and resin composite composition on composite adaptation to the cavity wall. Dental materials journal, 2014. 33(4): p. 499-503.

3. Cavalcante, L.M., et al., Influence of polymerization technique on microleakage and microhardness of resin composite restorations. Operative dentistry, 2003. 28(2): p. 200-206.

4. Cunha, L.G., et al., Effect of different photoactivation methods on the polymerization depth of a light-activated composite. Operative dentistry, 2003. 28(2): p. 155-159.

5. Campodonico, C.E., et al., Cuspal deflection and depth of cure in resin-based composite restorations filled by using bulk, incremental and transtooth-illumination techniques. The Journal of the American Dental Association, 2012. 142(10): p. 1176-1182.

6. Hafez, S., The Effect of Transtooth Curing Technique Using Different Led Light Intensities on Microhardness and Depth of Cure of Nanofilled Resin Composite Restorations. E.D.J. , 2018. 64(2): p. 1:7.

7. Manoranjan Reddy, Vanka Amit, and S. Prasad, Scanning Electron Microscopic Evaluation of Composite ResinDentin, Calcium Hydroxide-Dentin and Resin- Calcium Hydroxide Interfacial Gap with Composite Resin Restorations- An in vitro Study. People's Journal of Scientific Research 2011. 4(2): p. 41- 46.

8. Yaroub, M. and M.R. Hameed, Evaluation of marginal gap at the composite/enamel interface in Class II composite resin restoration by SEM after thermal and mechanical load cycling (An in vitro comparative study). Journal of baghdad college of dentistry, 2014. 26(4): p. 63-70.

9. Hardan, L.S., et al., Effect of different modes of light curing and resin composites on microleakage of Class II restorations-Part II. Odontostomatol Trop, 2009. 32(126): p. 29-37.

10. Bortolotto, T., F. Betancourt, and I. Krejci, Marginal integrity of resin composite restorations restored with PPD initiatorcontaining resin composite cured by QTH, monowave and polywave LED units. Dental materials journal, 2016. 35(6): p. 869-875. 